\title{
Feasibility of Full Percutaneous Segmental Stabilization of the Lumbar Spine With a Combination of an Expandable Interbody Cage and an Interspinous Spacer: Preliminary Results
}

\author{
RUDOLF MORGENSTERN, MD, PHD, CHRISTIAN MORGENSTERN, MD, PHD \\ Morgenstern Spine Institute, Centro Médico Teknon, Barcelona, Spain
}

\begin{abstract}
Introduction: We evaluated the feasibility of a full percutaneous approach with an expandable interbody cage and an interspinous spacer for a segmental stabilization of the anterior and posterior columns of the lumbar spine, respectively, with local anesthesia.

Methods: Patients were prospectively included between 2012 and 2018 in this single-center, feasibility case series. An expandable interbody cage was inserted with endoscopy-based, facet-sparing percutaneous transforaminal lumbar interbody fusion (pTLIF). An interspinous spacer was percutaneously placed through the same skin incision. Pre- and postoperative Visual Analog Scale (VAS) and Oswestry Disability Index (ODI) outcomes at 1, 3, 6, 12, and 24 months were obtained and evaluated with the Student $t$ test. Postoperative outcome was classified according to modified Macnab criteria.

Results: A total of 16 patients were included, presenting mean preoperative scores for VAS back of $6.9 \pm 2.5$, VAS leg $7.9 \pm 1.2$, and ODI $30.1 \pm 4.5$. Postoperative mean scores for VAS back of $1.9 \pm 2.1$, VAS leg 2.1 \pm 3.4 , and ODI $14.8 \pm 13.0$ significantly $(P<.001)$ decreased with a mean follow-up of $18.1 \pm 16.6$ months (range 1-65.2). Postoperative outcome was excellent and good for $13(81 \%)$ cases, fair for $2(13 \%)$, and poor for $1(6 \%)$ case with a preoperative spondylolisthesis, which required revision surgery due to persisting instability. Postoperative complications included 3 cases with transitory, ipsilateral dysesthesia and 2 cases with radiologic cage subsidence but no clinical symptoms. Median postoperative time until hospital discharge was 16 hours.

Conclusion: Our preliminary results for this full percutaneous technique show a similar outcome compared to conventional surgery with a fast patient recovery and early postoperative hospital discharge, opening the way to instrumented, outpatient surgery.
\end{abstract}

Endoscopic Minimally Invasive Surgery

Keywords: expandable interbody cage, interspinous spacer, percutaneous surgery, percutaneous transforaminal lumbar interbody fusion

\section{INTRODUCTION}

The purpose of instrumented minimally invasive spine surgery is to stabilize degenerative and/or pathologic levels in the lumbar spine less invasively than with conventional surgery. By using small skin incisions and avoiding wide tissue and bone dissection (ie, by progressive tissue dilatation), a similar outcome has been achieved with lower risk of bleeding and infection and less scar tissue formation, resulting in faster wound healing and early hospital discharge. ${ }^{1-4}$ As visual exposition is limited with minimally invasive spine surgery techniques, intraoperative fluoroscopy is usually required during surgery. Nonetheless, radiation can be progressively reduced once the learning curve has been mastered. ${ }^{5}$

Recently, endoscopy-based, facet-sparing percutaneous transforaminal lumbar interbody fusion (pTLIF) has been employed to place an interbody expandable device into the intervertebral disk through a small skin incision without tissue dissection and bone removal. ${ }^{6-10}$ Hence, this new application of the endoscopic, facet-sparing, transKambin approach offers a broad new array of possibilities with disruptive potential for spinal fusion surgery, opening the way to outpatient, instrumented spine surgery. ${ }^{10}$ Nonetheless, a poste- 

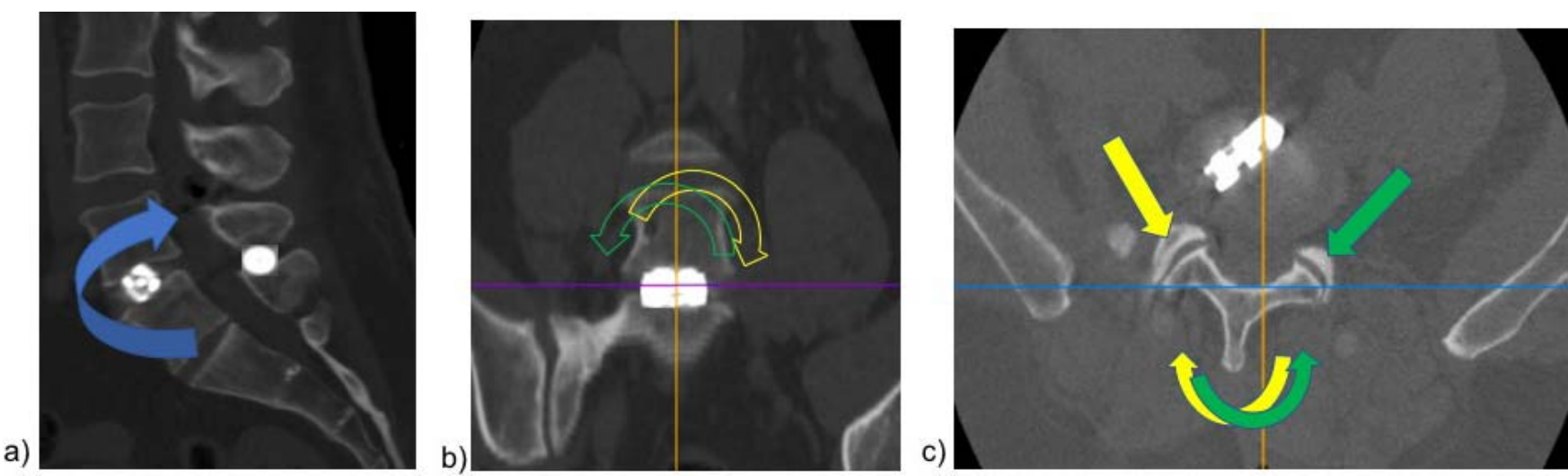

Figure 1. (a) In the sagittal plane, the interspinous implant limits segmental movement during extension (arrows) of the upper body by restricting movement of the posterior column of the operated level. (b) In the coronal plane, the interbody cage limits lateral bending (see arrows) in the operated level. (c) The percutaneous approach preserves the facets that restrict rotation (see arrows) of the operated level. Note how the interbody cage is correctly placed over the midline of the intervertebral disk in the axial plane.

rior fixation device is still required to achieve a $360^{\circ}$ segmental stabilization. Several percutaneous posterior fixation devices ${ }^{1-15}$ have been recently introduced that can be indicated, depending on the surgeon's preferences and the patient's demographic parameters, such as age, physical activity, gender, bone quality, adjacent segment conditions, ${ }^{4}$ and so on. Interspinous spacers ${ }^{11-13}$ have been used with varying success in the past decade. The clinical success correlated in most studies with an adequate preoperative indication. Hence, the success of the interspinous device seems to depend on the expected postoperative biomechanical demand by the patient and consequent stress that the construct will have to support. ${ }^{11-13}$

The aim of this case series was to evaluate the feasibility and preliminary clinical outcome of a full percutaneous combination of an expandable interbody cage and an interspinous spacer to stabilize the anterior and posterior columns, respectively, of the operated level of the lumbar spine (Figure 1). We hypothesized that a full percutaneous insertion of these 2 devices through a single, small skin incision under local anesthesia would provide sufficient stability to the operated level, resulting in a corresponding successful clinical outcome, and allow a rapid recovery of the patient. This novel technique could open the way to outpatient, instrumented surgery of the lumbar spine.

\section{METHODS}

\section{Case Series Design}

For this prospective case series, all patients were informed about the characteristics of the operation, difficulty, and potential complications. Written informed consent was obtained from all patients.

\section{Case Series Population}

Adults being surgically treated for low back pain and/or radiating leg pain between 2012 and 2018 in a private health care center were prospectively evaluated. Inclusion criteria comprised degenerative disc disease of the lumbar spine presenting with low back pain with or without uni- or bilateral radicular leg pain or neurogenic claudication and a corresponding alteration in the lumbar spine confirmed by magnetic resonance imaging, computed tomography scan, and/or lumbar spine X-rays. The preoperative assessment followed a standard protocol that includes preoperative magnetic resonance imaging/computer tomography scan of the lumbar spine, electromyogram of peripheral nerves, and additional dynamic $\mathrm{X}$-rays in cases with suspected instability of the lumbar spine. Careful physical examination was done to locate the affected level. Perioperative discography was systematically performed before the final decision to surgically target a level.

Exclusion criteria comprised patients suffering from absolute central stenosis without sitting pain release, high-grade vertebral instability and spondylolisthesis Meyerding grade > I, drug abuse, neoplasm, bone infection, and systemic diseases.

\section{General Versus Local Anesthesia}

Local anesthesia (bupivacaine and lidocaine solution at $1 \%$ ) with intravenous sedation, analgesia, and cardiopulmonary monitoring was per- 

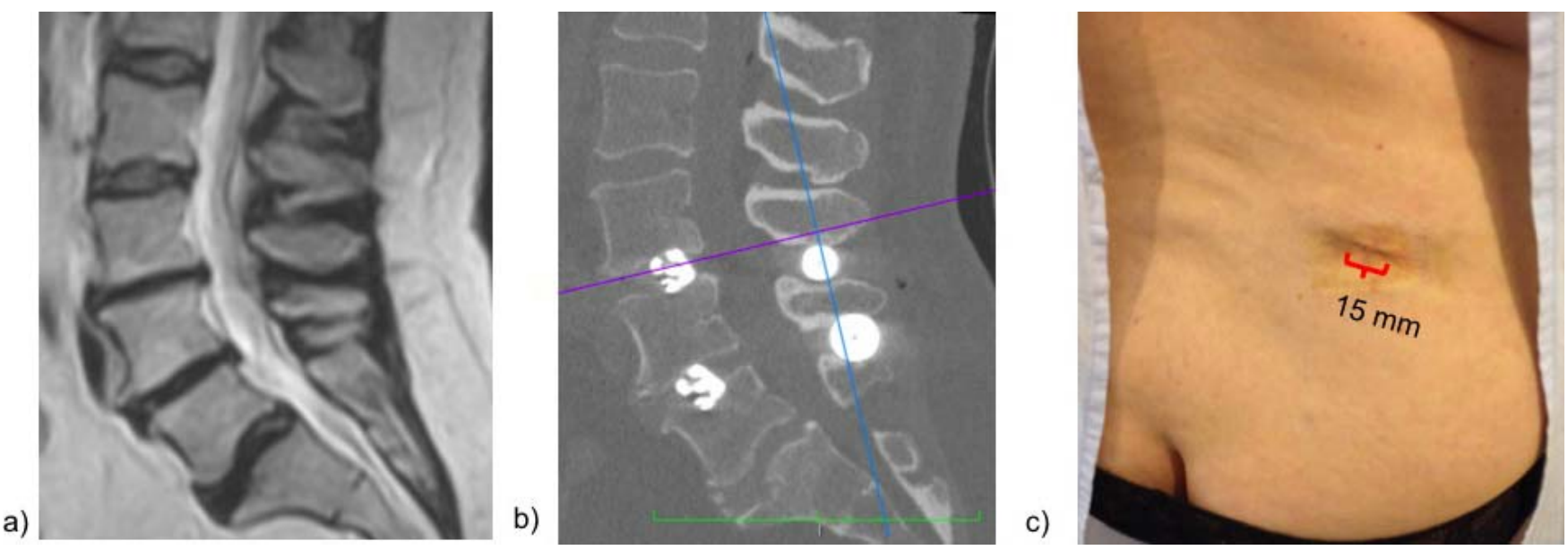

Figure 2. (a) Preoperative magnetic resonance imaging sagittal images of the lumbar spine of a female patient with degenerative disc disease and osteoarthritic changes (Modic II) at L3 to L4 and L4 to L5. (b) Postoperative computed tomography scan of the lumbar spine with an expandable cage and a percutaneous interspinous spacer at L3 to L4 and L4 to L5, respectively. (c) All 4 implants were percutaneously introduced through 1 single skin incision of $15 \mathrm{~mm}$.

formed by an independent anesthesiologist who was present during the whole surgical procedure. Local anesthesia was used on a patient's request, required written preoperative authorization by the anesthesist, and was used if the expected duration of the surgery was less than 2 hours. General anesthesia was complemented with neuromonitoring performed by an independent neurophysiologist. Somatosensory evoked potentials and motor evoked potentials were employed during the whole surgical procedure to monitor involved peripheral nerves.

\section{Surgical Technique}

The endoscopic, facet-sparing percutaneous transforaminal lumbar interbody fusion technique for the insertion of an expandable cage (Opticage, Interventional Spine Inc, Irvine, California; RISE, Globus Medical Inc, Audubon, Pennsylvania; Concorde Lift, Depuy-Synthes Inc, Raynham, Massachusetts) was performed as previously described ${ }^{9,10}$ under C-arm fluoroscopic control. Cage size was preoperatively measured with a preoperative magnetic resonance imaging/computer tomography scan. Specially designed access instrumentation (Optiport, Interventional Spine) was employed for progressive dilatation, optional foraminoplasty with manual reamers, and the facet-sparing, trans-Kambin (transforaminal) approach. ${ }^{9,10}$ No open soft tissue and bone dissection is usually required for this approach. Intraoperatively, the cage's size was confirmed with C-arm fluoroscopy after insertion of the cage. In cases where the cage's size needed to be modified after intradiscal placement, the cage was collapsed, removed, and replaced with a new cage with the optimal size. The cage's expansion height was determined by the surgeon using tactile feedback during the expansion of the cage in the intervertebral disk. During the cage's expansion, fluoroscopic control was employed to ensure no kyphotic deformity. In case a kyphotic deformity was detected, the cage was collapsed, repositioned, and reexpanded until correct sagittal balance was achieved. In all cases, fluoroscopic images in coronal and lateral view were recorded and archived. The interbody implant and remaining intervertebral disk space were filled with demineralized bone matrix to further stimulate intervertebral fusion.

In a second step, an interspinous spacer (RENEGADE, Globus Medical; APERIUS, Medtronic Inc, Fridley, Minnesota) was percutaneously placed through the same skin incision previously employed for placing the expandable cage. After progressive tissue dilatation, the spacer was placed between the spinous process of the affected and the caudal level under $\mathrm{C}$-arm fluoroscopic control as previously described $^{12,13}$ (Figure 2). Finally, the skin was closed with reabsorbing sutures. Early ambulation in the upright position is usually resumed on the same day of surgery.

\section{Outcome Evaluation and Follow-Up}

Pre- and postoperative outcome was quantitatively assessed at 1, 3, 6, 12, and 24 months after surgery. Lower back and radiating leg pain intensity were separately measured with the Visual Analog Scale (VAS), while disability was evaluated with the Oswestry Disability Index (ODI). ${ }^{16}$ Patient outcomes were classified as excellent, good, fair, and poor according to modified Macnab criteria. ${ }^{17}$ 
Table 1. Demographic and surgical characteristics of the case series.

\begin{tabular}{lc}
\hline Parameter & Value \\
\hline Age, y, mean \pm SD (range) & $70.3 \pm 10.3(51.6-88.5)$ \\
Male, no. (\%) & $3(18.8)$ \\
Revision surgery, no. (\%) & $4(25)$ \\
Preoperative clinical symptoms, no. (\%) & $15(94)$ \\
Back pain & $11(69)$ \\
Radiating leg pain & $5(31)$ \\
Neurogenic claudication & \\
Preoperative diagnosis ${ }^{\mathrm{a}}$, no. $(\%)$ & $5(31)$ \\
DDD with posterior impingement & $6(38)$ \\
DDD with foraminal stenosis & $5(31)$ \\
DDD with central stenosis & $2(13)$ \\
Adjacent segment degeneration & $4(25)$ \\
Spondylolisthesis (grade I) & \\
\hline
\end{tabular}

Abbreviation: DDD indicates degenerative disc disease.

${ }^{a}$ Multiple diagnoses per case may apply.

Computed tomography scans were taken in the immediate postoperative period (within 24 hours after surgery) to confirm correct implant placement. Additional radiological controls were performed at 4 weeks and 1 year after surgery.

\section{Statistical Analysis}

The Student $t$ test was employed to statistically compare the pre- and postoperative results. Statistical significance was defined at $P<.05$.

\section{RESULTS}

\section{Patient Demographic Characteristics}

A total of 16 cases were included in this case series. Demographics and preoperative diagnosis of the studied cohort are shown in Table 1.

Of the $4(25 \%)$ cases that had been previously operated on the lumbar spine (revision surgery cases), 1 case had undergone 3 endoscopic discectomies due to a recurrent foraminal herniation at $\mathrm{L} 3$ to $\mathrm{L} 4$, and 2 cases had been previously instrumented with transpedicular screws and rods from L3 to S1 and from L3 to L5, respectively, resulting in an adjacent segment syndrome. The last revision case had obtained interspinous spacers at L3 to L4 and L4 to L5 5 years previously and suffered from a foraminal stenosis at L4 to L5. In the latter case, only an expandable interbody cage was added to L4 to L5, while the interspinous spacers were maintained.

\section{Outcome and Results}

The surgical characteristics of the operated patients can be found in Table 2. Three (19\%) patients obtained an expandable interbody cage at 2 levels simultaneously during the same surgery, resulting in a
Table 2. Surgical characteristics of the operated patients.

\begin{tabular}{lc}
\hline Operated levels (interbody cage) & \\
L2/L3 & $2(11 \%)$ \\
L3/L4 & $5(26 \%)$ \\
L4/L5 & $11(58 \%)$ \\
L5/S1 & $1(5 \%)$ \\
Total levels & 19 \\
Operated levels (interspinous spacer) & \\
L2/L3 & $2(10 \%)$ \\
L3/L4 & $8(38 \%)$ \\
L4/L5 & $11(52 \%)$ \\
L5/S1 & 0 \\
Total levels & 21 \\
Anesthesia & $6(38 \%)$ \\
General anesthesia with neuromonitoring & $10(62 \%)$ \\
Local anesthesia with sedation &
\end{tabular}

total of 19 operated disks. A total of $6(38 \%)$ patients obtained an interspinous spacer at 2 levels simultaneously during the same surgery. One patient already had an interspinous spacer at 2 levels, and no additional spacers were added, resulting in a total of 21 operated levels with interspinous spacers.

The pre- and postoperative VAS back, VAS leg, and ODI scores are detailed in Table 3 for an overall mean follow-up of $18.1 \pm 16.6$ (range 1.0-65.2) months. Statistical significance $(P<.001)$ was found between the pre- and postoperative VAS back, VAS leg, and ODI scores. Outcomes according to modified Macnab criteria were $9(56 \%)$ excellent, $4(25 \%)$ good, $2(13 \%)$ fair, and $1(6 \%)$ poor result. The outcome is represented in Figure 3. Hence, a total of $13(81.3 \%)$ cases had an excellent and good outcome.

Median postoperative time until first ambulation/ walking was 4 hours (range 1.5-8 hours). Median postoperative time until hospital discharge was 16 hours (range 12-26 hours).

\section{Complications}

Transitory, ipsilateral dysesthesia was reported for $3(19 \%)$ cases, with paresthesia and radiating leg pain in the area of the dermatome corresponding to the operated level. All cases fully recovered with

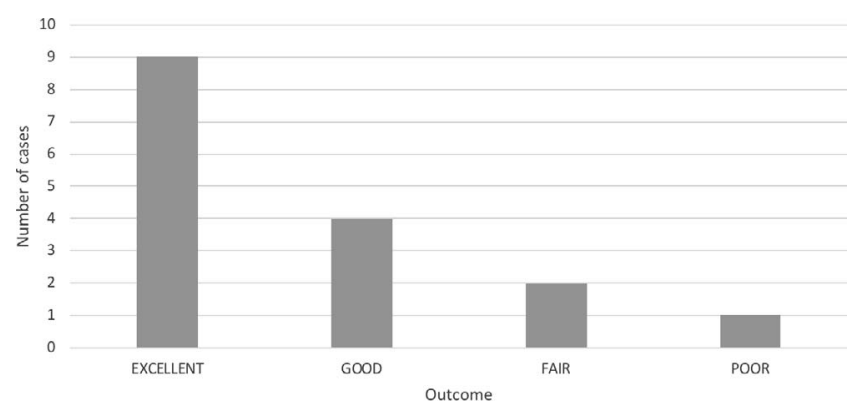

Figure 3. Outcome distribution according to modified Macnab criteria for 16 cases. 
Table 3. Pre- and postoperative Visual Analog Scale (VAS) and Oswestry Disability Index (ODI) scores. ${ }^{a}$

\begin{tabular}{|c|c|c|c|c|c|c|}
\hline & $\begin{array}{l}\text { Preoperative Score } \\
(\mathrm{n}=16)\end{array}$ & $\begin{array}{c}\text { Postoperative Score } \\
\text { at } 1 \text { mo } \\
(\mathrm{n}=16)\end{array}$ & $\begin{array}{c}\text { Postoperative Score } \\
\text { at } 12 \text { mo } \\
(\mathrm{n}=12)\end{array}$ & $\begin{array}{c}\text { Postoperative Score } \\
\text { at } 24 \text { mo } \\
(\mathrm{n}=7)\end{array}$ & $\begin{array}{c}\text { Latest Follow-Up } \\
\text { Postoperative Score } \\
(n=16)\end{array}$ & $P$ Value $^{\mathrm{c}}$ \\
\hline VAS back & $6.9 \pm 2.5(0-10)$ & $4.1 \pm 2.5(0-10)$ & $2.8 \pm 2.0(0-7)$ & $1.3 \pm 1.4(0-3)$ & $1.9 \pm 2.1(0-7)$ & $<.001$ \\
\hline VAS leg & $7.9 \pm 1.2(4-10)$ & $3.8 \pm 2.6(0-9)$ & $1.0 \pm 2.4(0-7)$ & $1.3 \pm 3.4(0-9)$ & $2.1 \pm 3.4(0-9)$ & $<.001$ \\
\hline ODI scores & $30.1 \pm 4.5(21-39)$ & $23.5 \pm 9.9(10-49)$ & $16.9 \pm 7.6(8-33)$ & $13.1 \pm 15(2-45)$ & $14.8 \pm 13.0(0-45)$ & $<.001$ \\
\hline
\end{tabular}

${ }^{\mathrm{a}}$ Scores are displayed as mean $\pm \mathrm{SD}$ (range).

${ }^{\mathrm{b}}$ For a mean follow-up of $18.1 \pm 16.6$ months.

${ }^{\mathrm{c}}$ Statistical significance of latest follow-up compared to preoperative scores.

oral pregabalin treatment after 4 to 6 weeks. No cases with paresia or muscle weakness were reported.

In $2(13 \%)$ cases, postoperative radiologic controls showed subsidence of an expandable cage. One case was an 89-year-old female with a history of severe osteoporosis who presented subsidence of an expandable cage into the inferior end plate of the superior vertebra as an incidental finding in the 4week routine X-ray control (Figure 4). The patient was and still is asymptomatic with current scores for VAS back of 0 , VAS leg 0 , and ODI 5 after a 6month follow-up. The second case was a 77-year-old female with a history of treatment for osteoporosis who presented a sudden postoperative increase in radiating leg pain after being pain free in the immediate postoperative control. Subsidence of the expandable cage was found at 2 weeks postoperative X-ray control. Nonetheless, the patient has fully recovered and shows no leg pain after a 3-month follow-up (VAS back and leg, 0; ODI, 1).

Two cases were classified as fair. The first was a 54-year-old female with a history of severe osteoporosis who presented a fractured L3 and L4 spinous process after a 3-year follow-up (Figure 5). She had been pain free until this incident was reported. The second case was a 64-year-old male operated with a cage at L4 to L5 and L5 to S1 and an interspinous spacer at L4 to L5 and transfacet screws at L5 to S1 due to advanced degenerative disc disease and osteochondrosis with Modic changes at L4 to L5 and L5 to S1. However, the postoperative clinical follow-up showed only a slight improvement, with persisting back and leg pain. Postoperative computed tomography and electromyogram control showed no pathologic evidence. We hypothesize that, in this case, the stability provided by the interspinous spacer may not be sufficient during load bearing and should probably be additionally stabilized with transpedicular screws and rods.

One case required revision surgery due to a displaced interspinous spacer that was detected in a routine radiographic control on an asymptomatic patient. The spacer was removed and replaced with another interspinous spacer at the same level. The postoperative outcome is excellent with scores for VAS back of 3 , VAS leg 0 , and ODI 19 with a 1 -year follow-up.

One case was classified as poor: a 62-year-old female with a preoperative grade I spondylolisthesis at L4 to L5 who required revision surgery due to persisting instability. Transpedicular screws and rods were percutaneously added as a posterior a)

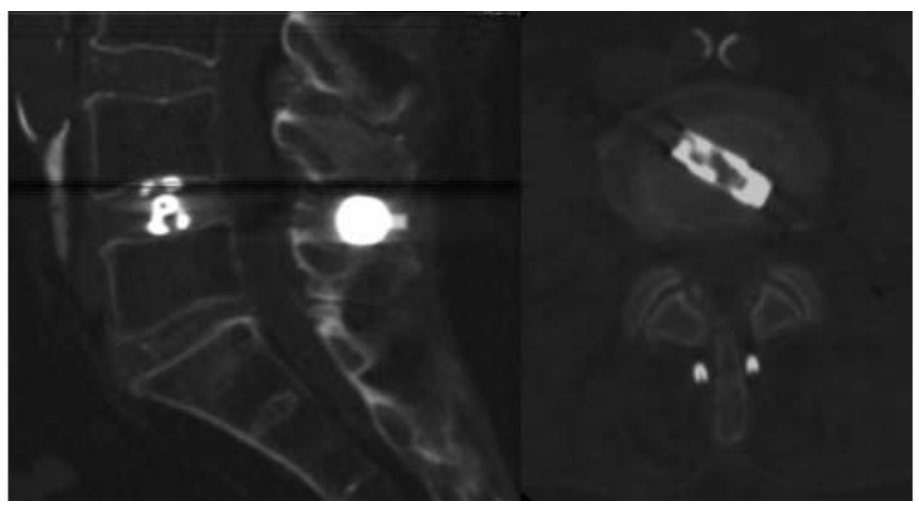

b)

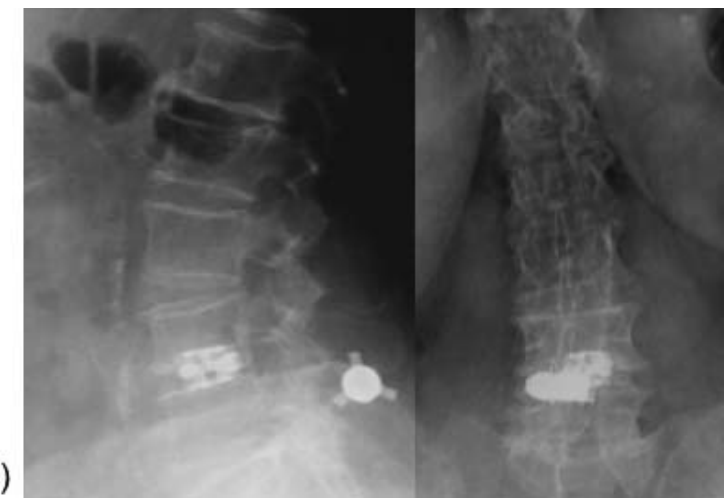

Figure 4. Incidental finding in the routine postoperative 4-week X-ray control (b) of subsidence of the expandable cage with osteointegration into the inferior end plate of the superior vertebra in comparison to the immediate postoperative computed tomography scan (a). The patient has been clinically asymptomatic and reports no pain. 


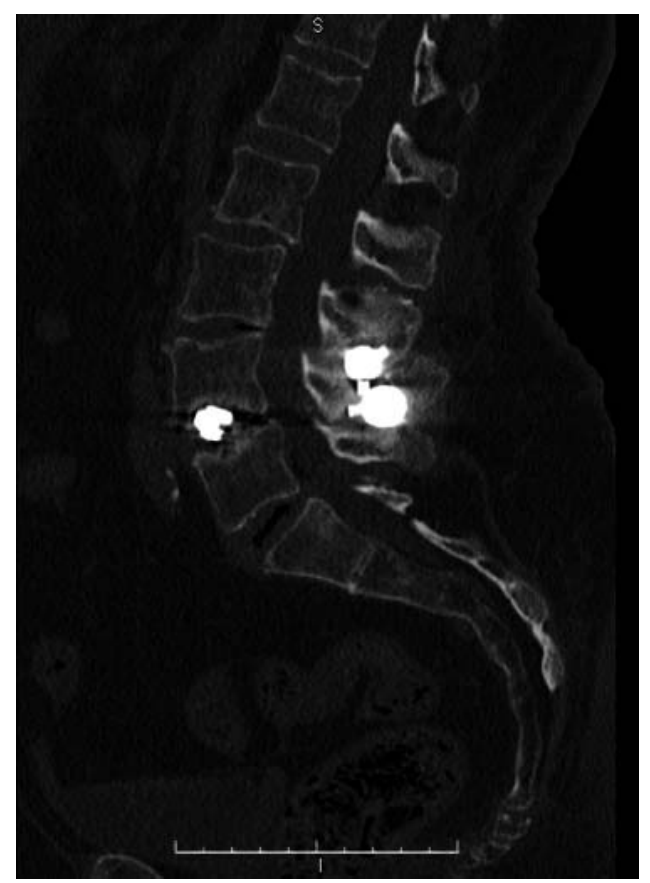

Figure 5. Female patient with severe osteoporosis presenting fractured L3 and L4 spinous processes after a 3-year follow-up.

fixation device without removing the existing interspinous spacer. Follow-up was lost after 3 months, and the outcome of this case was classified as poor.

No cases of infection, dural tear, pseudoarthrosis, and nonunion have been reported.

\section{DISCUSSION}

In this case series, we have demonstrated the clinical feasibility of a combination of an expandable interbody device and a percutaneous interspinous spacer for a full percutaneous instrumented segmental stabilization of the lumbar spine. Both implants were percutaneously inserted through a single skin incision of approximately $15 \mathrm{~mm}$ length (Figure 2), and most patients were operated on under local anesthesia and sedation. The expandable interbody cage stabilizes the anterior column of the operated level in the lumbar spine and allows increasing the disk's height through its expansion. If the expandable cage is placed correctly over the midline of the intervertebral disk (Figure 1c), it will limit lateral bending (Figure 1b) and support axial compression on the anterior column of the lumbar spine during forward flexion of the upper body. On the other hand, the interspinous spacer limits lumbar spine movement during upper body extension by stabi- lizing the posterior column of the operated level of the lumbar spine (Figure 1a). Due to the full percutaneous approach, bone and tissue removal is avoided. Hence, natural stabilization structures, such as the facets, the ligamentum flavum, and the annulus, are preserved. The facets are structures that limit spinal rotation and translation. Hence, the combination of the expandable cage, the interspinous spacer, and the preserved facets, annulus, and ligamentum flavum provide further stability to the operated segment (Figure 1c). However, the biomechanical stability obtained is not on par with that obtained by full instrumentation of the lumbar spine (ie, with transpedicular rods and screws). Especially shear forces in the anterior/posterior plane, as in an unstable spondylolisthesis, cannot be properly addressed with this percutaneous technique. Despite studies ${ }^{11}$ showing strong evidence for interspinous spacers in cases with grade I spondylolisthesis, this technique should be carefully indicated in cases of vertebral instability. Preoperative assessment with functional $\mathrm{X}$-rays of the lumbar spine is strongly recommended. In cases of a radiologically confirmed, unstable spondylolisthesis, this technique should not be employed.

This novel, facet-sparing technique for instrumented stabilization of the lumbar spine is intended not to replace existing surgical techniques (ie, endoscopic foraminoplasty or instrumented surgery with transpedicular rods and screws) but rather to complement present surgical options, as it provides a compromise between endoscopic foraminoplasty and full-scale, instrumented spine surgery with transpedicular screws and rods. In fact, many of the presented cases here required foraminoplasty with manual reamers previous to inserting the cage. As seen with the case with a poor outcome, instrumented revision surgery with transpedicular screws and rods is possible and relatively easy to implement. In our opinion, the presented full percutaneous approach can be very useful if carefully indicated, as it allows instrumenting the lumbar spine and stabilizing the operated level with the advantages of full percutaneous surgery (ie, a small skin incision, no bone and tissue removal, and local anesthesia). In our opinion, this technique is of interest for patients with low physical activity who do not wish to undergo extensive lumbar spine surgery and may profit from target-oriented, ${ }^{18}$ outpatient percutaneous spine surgery. Patients over 
50 years old with limited and not too strenuous daily physical activity should be appropriate candidates for this technique. It should not be indicated in young or physically active patients. Lumbar pathologies at L5 to $\mathrm{S} 1$ are also difficult to address with this technique due to the intrinsic limitation of the $\mathrm{S} 1$ spinous process, which is usually too small to support an interspinous spacer. However, cases with a pathology at L5 to S1 can be instrumented with percutaneous transfacet screws or percutaneous transpedicular screws and rods. ${ }^{9,10}$

Most of the described complications were related to osteoporotic bone that provided insufficient support to the implanted devices, resulting in cage subsidence and fracture of a spinous process. However, subsidence should be differentiated, depending on its stability. Unstable subsidence usually results in pseudoarthrosis. However, no pseudoarthrosis has been reported in our case series so far. Stable subsidence usually results in osteointegration of the cage with the end plate. The 2 described cases with subsidence in our cohort were clinically asymptomatic and radiologically stable in the follow-up. Nonetheless, this technique should be carefully indicated or even contraindicated in cases with severe osteoporosis. Extensive preoperative assessment of a patient's bone quality (eg, with bone densitometry) is strongly recommended. Finally, the rate of $19 \%$ cases reporting postoperative transient dysesthesia is similar to other studies ${ }^{9,10}$ employing the endoscopic, trans-Kambin approach to place an expandable cage in the intervertebral disk, and, as expected, all cases fully resolved within a few weeks.

All limitations of a small pilot case series apply here: our preliminary results require confirmation in a multicenter study with an extensive cohort and longer-term follow-up. It was not the intention of this pilot study to evaluate the fusion rate of the operated levels in this case series. Radiologic evidence of interbody fusion needs to be evaluated with a long-term mean follow-up.

\section{CONCLUSION}

We have presented preliminary results of a novel, full percutaneous, facet-sparing technique for instrumented, segmental stabilization of the lumbar spine with local anesthesia through a single, small skin incision. This technique is intended to complement present surgical techniques, as it represents another minimally invasive surgical option before full-scale instrumented spine surgery with transpedicular screws and rods. Our outcome does not differ from conventional minimally invasive spine or open surgery, but patient recovery was faster, allowing early hospital discharge within 16 hours after surgery. If carefully indicated, our results should be confirmed in a larger study, opening the way to ambulatory, instrumented surgery of the lumbar spine in an outpatient facility.

\section{REFERENCES}

1. Shunwu F, Xing Z, Fengdong Z, Xiangqian F. Minimally invasive transforaminal lumbar interbody fusion for the treatment of degenerative lumbar diseases. Spine (Phila $\mathrm{Pa}$ 1976). 2010;35(17):1615-1620.

2. Kim JS, Jung B, Lee SH. Instrumented minimally invasive spinal-transforaminal lumbar interbody fusion (MIS-TLIF): minimum 5-years follow-up with clinical and radiologic outcomes. Clin Spine Surg. 2018;31(6):E302-E309.

3. Karikari IO, Isaacs RE. Minimally invasive transforaminal lumbar interbody fusion: a review of techniques and outcomes. Spine (Phila Pa 1976). 2010;35(26):S294-S301.

4. Ruetten S, Komp M, Merk H, et al. Fullendoscopic interlaminar and transforaminal lumbar discectomy versus conventional microsurgical technique: a prospective, randomized, controlled study. Spine (Phila Pa 1976). 2008;33(9):931939.

5. Morgenstern R, Morgenstern C, Yeung AT. The learning curve in foraminal endoscopic discectomy: experience needed to achieve a 90\% success rate. Int J Spine Surg. 2007;1(3):100-107.

6. Morgenstern R. Full endoscopic TLIF approach with percutaneous posterior transpedicular screw fixation in a case of spondylolisthesis grade I with L4-L5 central stenosis. J Crit Spine Cases. 2010;3:115-119.

7. Syed H, Voyadzis JM. True percutaneous transforaminal lumbar interbody fusion: case illustrations, surgical technique, and limitations. J Neurol Surg A Cent Eur Neurosurg. 2016;77(4):344 353.

8. Morgenstern $\mathrm{R}$, Morgenstern $\mathrm{C}$, Jané $\mathrm{R}$, Lee $\mathrm{SH}$. Usefulness of an expandable interbody spacer for the treatment of foraminal stenosis in extremely collapsed disks: preliminary clinical experience with the endoscopic posterolateral transforaminal approach. J Spinal Disord Tech. 2011;24(8):485-491.

9. Morgenstern R, Morgenstern C. Percutaneous transforaminal Lumbar interbody fusion (pTLIF) with a posterolateral approach for the treatment of degenerative disk disease: feasibility and preliminary results. Int J Spine Surg. 2015;9:41.

10. Morgenstern R, Morgenstern C. New implants and techniques in minimally invasive spine surgery: true percutaneous transforaminal lumbar interbody fusion (pTLIF) with the posterolateral transforaminal endoscopic approach. In: Delfini $\mathrm{R}$ et al, eds. Modern Thoraco-Lumbar Implants for Spinal Fusion. New York: Springer-Verlag; 2018:69-91.

11. Davis R, Auerbach J, Bae H, Errico TJ. Can low-grade spondylolisthesis be effectively treated by either coflex interlaminar stabilization or laminectomy and posterior spinal fusion? Two-year clinical and radiographic results from the randomized, prospective, multicenter US investigational device 
exemption trial, spondylolisthesis treated with coflex stabilization of fusion. J Neurosurg Spine. 2013;19(2):174-184.

12. Zhao H, Duan LG, Gao YS, et al. Comparison of two FDA-approved interspinous spacers for treatment of lumbar spinal stenosis: superion versus X-STOP-a meta-analysis from five randomized controlled trial studies. J Orthop Surg Res. 2018;13(1):42.

13. Tapp SJ, Martin BI, Tosteson TD, et al. Understanding the value of minimally invasive procedures for the treatment of lumbar spinal stenosis: the case of interspinous spacer devices. Spine J. 2018;18(4):584-592.

14. Powers CJ, Podichetty VK, Isaacs RE. Placement of percutaneous pedicle screws without imaging guidance. Neurosurg Focus. 2006;20(3):E3.

15. Chin KR, Seale J, Cumming V. Mini-open or percutaneous bilateral lumbar transfacet pedicle screw fixation: a technical note. J Spinal Disord Tech. 2015;28(2):61-65.

16. Fairbank, Jeremy CT, Pynsent, PB. The Oswestry Disability Index. Spine (Phila Pa 1976). 2000;25(22):29402953.

17. Macnab I. Negative disc exploration: an analysis of the causes of nerve-root involvement in sixty-eight patients. $J$ Bone Joint Surg Am. 1971;53(5):891-903.

18. Morgenstern R, Morgenstern C. Assessment and selection of the appropriate individualized technique for endoscopic lumbar disc surgery. In: Menchetti PP, ed. Minimally Invasive Surgery of the Lumbar Spine. New York: Springer-Verlag; 2014:107-120.

Disclosures and COI: The first author reports royalty fees from DePuy/Synthes and Interventional Spine Inc, and royalty fees from Joimax $\mathrm{GmbH}$ outside of the submitted work. The second author reports no conflict of interests.

Corresponding Author: Dr Christian Morgenstern, Morgenstern Spine Institute, Centro Médico Quirón Teknon, C/Vilana 12, 08022 Barcelona, Spain. Phone: 349325318 67; Email: morgenstern@dr.teknon.es.

Published 21 December 2018

This manuscript is generously published free of charge by ISASS, the International Society for the Advancement of Spine Surgery. Copyright $\odot 2018$ ISASS. To see more or order reprints or permissions, see http://ijssurgery.com. 